\title{
MULTICOMUTAÇÃO E AMOSTRAGEM BINÁRIA EM ANÁlISE QUíMICA EM FLUXO. DETERMINAÇÃO ESPECTROFOTOMÉTRICA DE ORTOFOSFATO EM ÁGUAS NATURAIS
}

Eloisa Ap. Mocheuti Kronka, Boaventura Freire dos Reis*

Centro de Energia Nuclear na Agricultura - Universidade de São Paulo - Av. Centenário, 303, CP 96 - $13400-970$ - Piracicaba - SP Jonas A. Vieira, Telma Blanco e Ana Paula G. Gervasio

Instituto de Química de São Carlos - USP

Recebido em 20/6/96; aceito em 25/11/96

\begin{abstract}
MULTICOMMUTATION AND BINARY SAMPLING IN CHEMICAL FLOW ANALYSIS. SPECTROPHOTOMETRIC DETERMINATION OF PHOSPHATE IN NATURAL WATERS. A flow system based on multicommutation and binary sampling process was developed to implement the sample zone trapping technique in order to increase the spectrophotometric analytical range and to improve sensitivity. The flow network was designed with active devices in order to provide facilities to determine sequentialy analyte with a wide concentration range, employing a single pumping channel to propel sample and reagents solutions. The procedure was employed to determine ortophosphate ions in water samples of river and waste. Profitable features such as an analytical throughput of 60 samples determination per hour, relative standard deviation (r.s.d.) of $2 \%(\mathrm{n}=6)$ for a typical sample with concentration of $2.78 \mathrm{mg} / \mathrm{L}$ were achieved. By applying the paired $t$-test no significant difference at $95 \%$ confidence level was observed between the results obtained with the proposed system and those of the usual flow injection system.
\end{abstract}

Keywords: multicommutation; flow analysis; binary sampling; phosphate determination.

\section{INTRODUÇÃO}

O processo de análise química por injeção em fluxo (FIA) foi proposto em $1975^{1}$. Ao longo destes anos tem demonstrado grande sucesso, considerando-se que mais de 4.000 artigos já foram publicados por pesquisadores de vários países ${ }^{2,3}$.

A grande aceitação deste processo de análise química devese a simplicidade da instrumentação e a versatilidade do módulo de análise, possibilitando a implemenção de procedimentos analíticos automáticos, utilizando instrumentos de baixo custo. Neste sistema, as condições de reação podem ser facilmente controladas e repetidas. Além disso, as reações químicas ocorrem em um ambiente fechado, o que é uma vantagem adicional, pois diminui os riscos de contaminação.

A análise química por injeção em fluxo tem como conceito básico a introdução da solução da amostra em um fluido carregador, que a transporta para o detector. Ao longo do percurso analítico pode receber reagentes e passar por etapas de processamentos, tais como: extração líquido-líquido ${ }^{4}$; pré-concentração ${ }^{5}$; geração de fase gasosa para separar o analito ${ }^{6}$; diluição em linha ${ }^{7}$; diálise por membrana permeável a gás ${ }^{8}$; etc.

A introdução reprodutível da alíquota da solução da amostra no fluido carregador, é um dos fatores básicos para garantir bom desempenho dos sistemas FIA. Nos primeiros trabalhos, foi empregada seringa hipodérmica ${ }^{9}$ e, com a evolução do processo, outros dispositivos foram propostos para esta finalidade, sendo empregados, em maior escala, o injetor comutador ${ }^{10}$ e as válvulas rotativas de 6 e 8 vias ${ }^{11,12}$.

As válvulas solenóides de três vias também têm sido empregadas para este propósito, as quais oferecem como vantagem a possibilidade de um acionamento individual e sequencial, enquanto que os demais dispositivos possuem comutação solidária $^{13}$. O emprego destas válvulas permite que se altere facilmente o funcionamento do módulo de análise sem alterar a configuração do mesmo.

A multicomutação em sistema FIA associada à amostragem binária foi introduzida recentemente ${ }^{14}$ e possibilita manipular, com boa precisão, volumes da ordem de $5 \mu \mathrm{L}$. Este processo foi empregado para desenvolver um procedimento para a determinação sequencial de duas espécies químicas de interesse, utilizando o mesmo percurso analítico ${ }^{15}$.

O conceito básico de multicomutação e amostragem binária é a inserção sequencial, no percurso analítico, de pequenas alíquotas de amostra e dos reagentes. Assim, a reação química para produzir o composto a ser detectado, tem início durante a amostragem. Nos sistemas FIA usuais, o reagente é adicionado à amostra após a inserção no percurso analítico e portanto o intervalo de tempo da amostragem não é aproveitado para o desenvolvimento da reação. Em princípio, em um módulo de análise baseado no conceito de amostragem binária, tem-se um melhor aproveitamento do tempo, o que pode resultar em aumento da velocidade analítica.

O módulo de análise para implementar a amostragem binária, é constituído por um conjunto de válvulas solenóides de três vias, configuradas para efetuar a introdução da amostra e dos reagentes no percurso analítico, formando, a partir do ponto de inserção, uma sequência binária. Pequenas frações das soluções da amostra e do reagente são inseridas, alternadamente, no percurso analítico. O tempo de acionamento das válvulas e a vazão de bombeamento determinam o volume de cada alíquota. A inserção das soluções da amostra e dos reagentes no percurso analítico pode ser manipulada, empregando-se apenas um canal de bombeamento. Em um sistema FIA usual é necessário um canal de bombeamento para cada solução.

Em sistemas FIA, não é necessário completar a reação para se obter medidas com boa precisão. Esta característica pode ser empregada para ajustar a magnitude do sinal analítico à faixa operacional do detector, desde que o módulo de análise proporcione meios para este ajuste. Isto pode ser facilmente conseguido, empregando-se procedimentos analíticos baseados no processo de multicomutação ${ }^{14}$. Entretanto, quando a amostra é muito diluida, é necessário que a reação se complete, para que se possa alcançar um melhor limite de detecção. Quando fato desta natureza ocorre e a reação química possui cinética 
lenta, deve-se aumentar o intervalo de tempo de residência da zona da amostra no percurso analítico. Um dos recursos usados para esta finalidade é a técnica de parada de fluxo ${ }^{16,17}$ (stopped flow).

A determinação de ortofosfato em águas de rios é muito importante em estudos envolvendo a qualidade da água, sendo que a faixa de concentração varia dependendo do índice de poluição. $\mathrm{O}$ método de determinação baseado na reação com molibdato de amônio e redução com ácido ascórbico ou cloreto estanoso, em geral, é o mais utilizado ${ }^{18,19}$. Um intervalo de tempo, superior a $2 \mathrm{~min}$, é necessário para completar a reação, portanto, para amostras muito diluidas, o módulo de análise deve proporcionar meios para que o tempo de residência da amostra no percurso analítico seja desta ordem.

Recorrendo-se aos recursos oferecidos pelo processo de multicomutação e amostragem binária, pretende-se desenvolver um módulo de análise em que se possa ajustar, por "software", o tempo de residência da amostra no percurso analítico. Assim, associando-se esta facilidade à cinética de reação do íon ortofosfato com molibdato de amônio, pretende-se desenvolver um procedimento automático, para determinar este analito em uma ampla faixa de concentrações. Espera-se determinar ortofosfato em águas naturais e águas poluídas com resíduos domésticos, sem modificar a estrutura do módulo de análise.

\section{PARTE EXPERIMENAL}

\subsection{Soluções de reagentes e amostras}

Todas as soluções foram preparadas a partir de reagentes de grau analítico usando água destilada-desionizada.

Solução de molibdato de amônio $1,0 \%(\mathrm{~m} / \mathrm{v})$ foi preparada, dissolvendo-se 5,0 g do composto em $500 \mathrm{~mL}$ de água.

Solução de ácido ascórbico $1,0 \%(\mathrm{~m} / \mathrm{v})$ foi preparada, dissolvendo-se 1,0 g do composto em $100 \mathrm{~mL}$ de água. Esta solução foi preparada diariamente.

Solução estoque de ortofosfato $1000 \mathrm{mg} / \mathrm{L}$ foi preparada, dissolvendo-se $1,098 \mathrm{~g}$ de $\mathrm{KH}_{2} \mathrm{PO}_{4}$ em $250 \mathrm{~mL}$ de água.

Soluções padrão de ortofosfato de 0,$10 ; 0,20 ; 0,40 ; 0,60$; 0,80 e $1,00 \mathrm{mg} / \mathrm{L}$ e de 1,$00 ; 2,00 ; 4,00 ; 6,00 ; 8,00$ e $10,00 \mathrm{mg} /$ $\mathrm{L}$ e $0,014 \mathrm{~mol} / \mathrm{L} \mathrm{em} \mathrm{HNO}_{3}$, foram preparadas a partir da solução estoque de $1000 \mathrm{mg} \mathrm{PO}_{4}{ }^{3-} / \mathrm{L}$.

As amostras de água foram coletadas em garrafas de polietileno e acidificadas, adicionando-se $1 \mathrm{~mL}$ de ácido nítrico concentrado, para ter concentração final em torno de $0,014 \mathrm{~mol} / \mathrm{L}$. A determinação do analito foi efetuada no dia da coleta. Foram selecionadas 02 áreas de coleta, sendo uma ao longo do rio Piracicaba a partir do ponto de despejo dos resíduos domésticos da cidade e a outra a partir do ponto de descarte de efluentes de uma usina de açúcar e álcool da região.

\subsection{Equipamentos}

Espectrofotômetro Femto 432 equipado com cela de fluxo com volume interno de $180 \mu \mathrm{L}$ e passo ótico de $13 \mathrm{~mm}$.

Microcomputador 386, equipado com uma interface PCL 711 - S (American Advantech Corp).

Bomba peristáltica Ismatec, modelo MP $13 \mathrm{R}$, equipada com tubos de tygon e interface para controle externo de rotação por "loop" de corrente.

Interfaces eletrônicas para controle da velocidade da bomba peristáltica, sincronização da amostragem com a pulsação da bomba e acionamento das válvulas solenóides ${ }^{14}$

Módulo de análise constituído por 5 válvulas solenóides de 3 vias, bobinas de reação feitas de tubo de polietileno, tendo cada uma $100 \mathrm{~cm}$ de comprimento e diâmetro interno de $0,8 \mathrm{~mm}$.

\section{Descrição do módulo de análise.}

O módulo de análise foi desenvolvido, levando-se em consideração as características da reação química envolvida e o diagrama de fluxo é mostrado na figura 1. Inicialmente, todas as válvulas estão desligadas e o fluido carregador está passando continuamente pelo percurso analítico, através de $\mathrm{B}_{1}$. $\mathrm{O}$ ciclo de análise inicia-se quando o microcomputador aciona as válvulas $\mathrm{V}_{1}, \mathrm{~V}_{2}, \mathrm{~V}_{3}$ e $\mathrm{V}_{4}$, de acordo com o diagrama de tempo mostrado nesta figura. Estas válvulas ao serem acionadas, permitem a introdução de pequenas frações das soluções da amostra e dos reagentes no percurso analítico, formando a partir do ponto de junção $(\mathbf{x})$, uma sequência ternária, que corresponde às alíquotas das soluções da amostra, do molibdato de amônio e do ácido ascórbico $\left(\mathrm{R}_{1}, \mathrm{~A}, \mathrm{R}_{2}\right)$, conforme indicado no modelo da figura 2 .

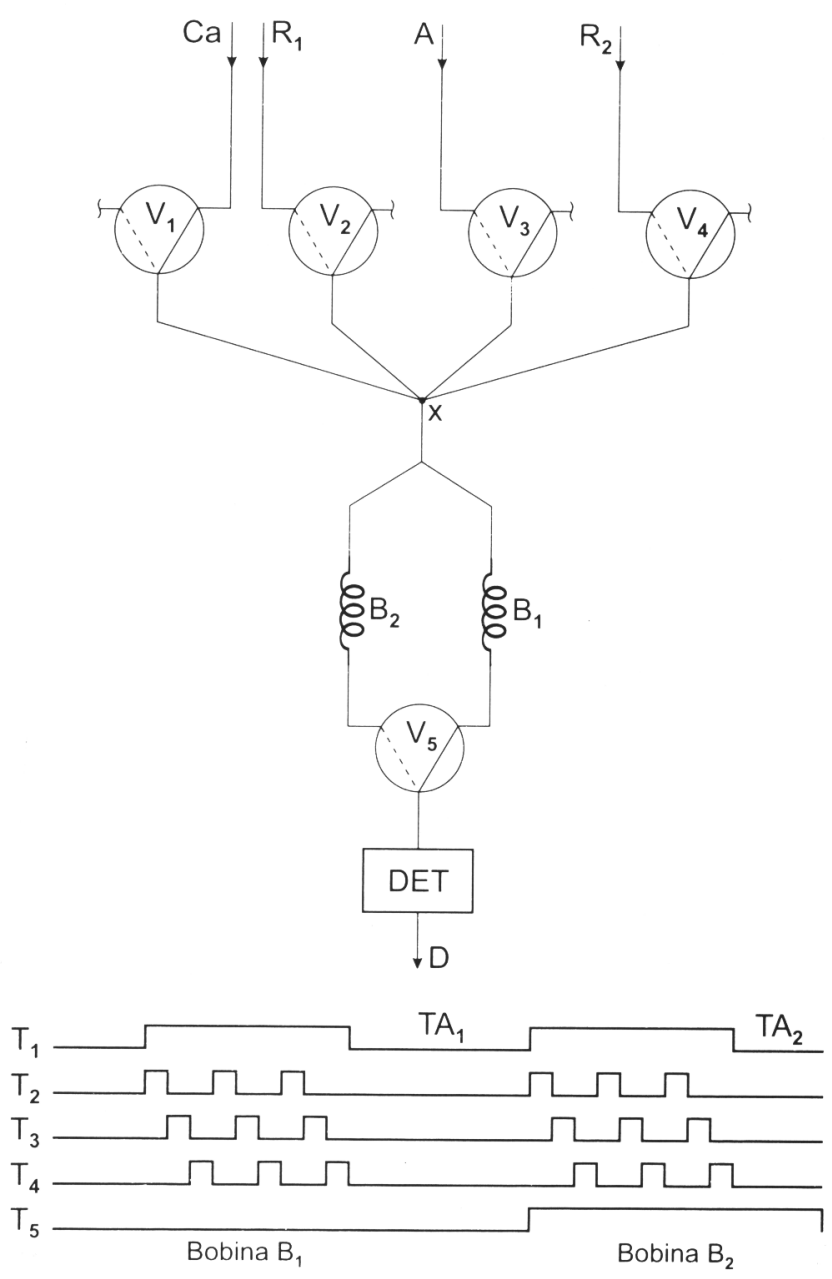

Figura 1. Diagrama de fluxo do módulo de análise. Ca = carregador da amostra; $A=$ amostra; $R_{1}=$ molibdato de amônio $1,0 \%(\mathrm{~m} / \mathrm{v}) ; R_{2}$ = ácido ascórbico $1,0 \%(\mathrm{~m} / \mathrm{v}) ; B_{1}$ e $B_{2}=$ bobinas de reação de tubo de polietileno, $100 \mathrm{~cm}$ de comprimento, $0,8 \mathrm{~mm}$ de diametro interno; DET = espectrofotômetro $(640 \mathrm{~nm}) ; D=$ descarte; $V_{1}, V_{2}, V_{3}, V_{4}$ e $V_{5}$ $=$ válvulas solenóides de 3 vias $. T_{1}, T_{2}, \ldots, T_{5}=$ intervalos de tempo de acionamento das válvulas $V_{1}, V_{2}, \ldots, V_{5} ; T_{A 1}$ e $T_{A 2}=$ intervalos de tempo para leitura dos sinais correspondente às zonas de amostras dos percurso analíticos $B_{1}$ e $B_{2}$, respectivamente.

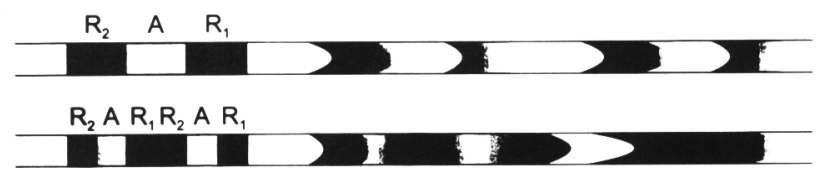

Figura 2. Modelo da dispersão mútua na interface das alíquotas das soluções. Em a, $A, R_{1}$ e $R_{2}=$ alíquotas da solução da amostra, molibdato de amônio e ácido ascórbico, respectivamente, representando um ciclo de amostragem. Em b, é representado dois ciclos de amostrtagem efetuados na mesma sequência de a, sendo o volume de cada alíquota metade do volume do caso anterior. 
A implementação de um sistema com estas características, requer o emprego de um programa dedicado, desenvolvido considerando-se as características da reação química envolvida e do módulo de análise. O programa para executar este processo, foi escrito em Quick BASIC 4.5, seguindo a lógica de execução mostrada na figura 3. Quando o mesmo é inicializado, o microcomputador solicita os valores correspondentes às variáveis de controle: duração dos acionamentos das válvulas envolvidas no ciclo de inserção; número de repetições do ciclo de inserção; número de replicatas; vazões de bombeamento; e o intervalo de tempo de residência da zona da amostra em cada bobina de reação $\left(\mathrm{B} 1, \mathrm{~B}_{2}\right)$. Além disso, pergunta se o início da etapa de amostragem, deve ser sincronizado com a pulsação da bomba peristáltica ${ }^{14}$. Uma vez fornecidos estes dados, o microcomputador assume o controle do processo analítico, efetuando a sequência de acionamentos das válvulas de acordo com o diagrama de tempo mostrado na figura 1 .

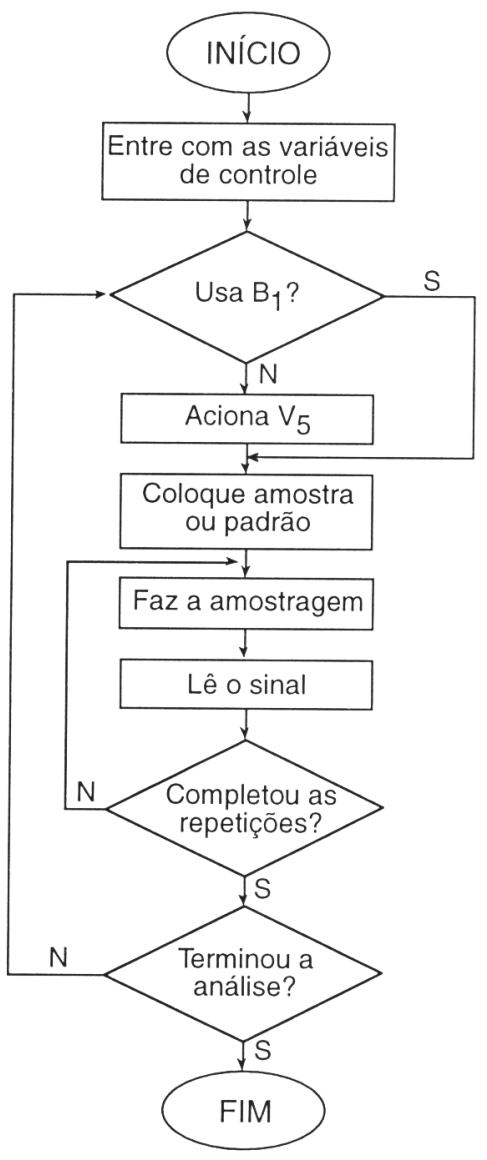

Figura 3. Fluxograma do "software" controle do módulo de análise.

O volume de cada fração das soluções inserido no percurso analítico é proporcional à vazão de bombeamento e ao tempo de acionamento das respectivas válvulas. O número destas frações define o volume da alíquota das soluções da amostra e dos reagentes utilizados em cada ciclo de análise.

Uma vez terminada a etapa de inserção das alíquotas das soluções da amostra e dos reagentes, espera-se um intervalo de tempo pré-determinado para que a zona da amostra esteja contida na bobina $B_{1}$. Em seguida, aciona-se a válvula $V_{5}$, desviando o fluxo carregador $\left(\mathrm{C}_{\mathrm{a}}\right)$ através da bobina $\mathrm{B}_{2}$ e, em consequência, a zona da amostra permanece em repouso na bobina $B_{1}$.

Mantendo-se a válvula $\mathrm{V}_{5}$ ligada e acionando-se, novamente, as válvulas $\mathrm{V}_{1}, \mathrm{~V}_{2}, \mathrm{~V}_{3}$ e $\mathrm{V}_{4}$, obedecendo a sequência anterior, insere-se na bobina $\mathrm{B}_{2}$ uma zona da amostra e reagentes semelhante ao descrito para o ciclo de amostragem anterior. Desligando-se estas válvulas e mantendo $\mathrm{V}_{5}$ acionada, a solução carregadora transporta esta mistura para a cela de fluxo, onde é efetuada a detecção espectrotofométrica. Após o registro do sinal, desliga-se a válvula $V_{5}$, a solução carregadora volta a fluir através da bobina $B_{1}$. A zona da amostra que estava em repouso nesta bobina, é transportada para a cela de fluxo, onde é efetuada a detecção. Nesta etapa, a velocidade de rotação da bomba peristáltica foi aumentada de 2 para $3 \mathrm{~mL} / \mathrm{min}$, para diminuir o intervalo de tempo necessário ao deslocamento da zona da amostra para o descarte (D). Em seguida, é restabelecida a vazão inicial ( $2 \mathrm{~mL} / \mathrm{min})$ e as válvulas $\mathrm{V}_{1}, \mathrm{~V}_{2}, \mathrm{~V}_{3}$ e $\mathrm{V}_{4}$ são acionadas novamente, iniciando-se outro ciclo de análise.

Empregando esta estratégia, obtém-se duas leituras da mesma amostra, cuja diferença em magnitude é função do tempo de residência da zona da amostra no percurso analítico estabelecido em cada ciclo de análise. Então, para estudar o efeito do tempo de repouso da zona da amostra no desenvolvimento da reação, o tempo de residência da amostra em $B_{2}$ foi mantido em $50 \mathrm{~s}$ e variou-se o tempo de residência em $\mathrm{B}_{1}$ de 50 a $160 \mathrm{~s}$

Ao terminar o número de replicatas estabelecido, o microcomputador pergunta se deseja trocar de amostra, caso afirmativo, aciona as válvulas $\mathrm{V}_{1}$ e $\mathrm{V}_{2}$ pelo tempo programado para trocar de amostra, inciando em seguida novo ciclo analítico.

\section{RESULTADOS E DISCUSSÃO}

A formação do ácido molibdofosfórico e sua redução com ácido ascórbico requer um intervalo de tempo em torno de $140 \mathrm{~s}$ para completar a reação ${ }^{18,19}$. Em sistemas FIA este intervalo de tempo pode ser considerado longo, pois o tempo de residência da amostra no percurso analítico, em geral, é menor que 60 segundos. Entretanto, quando a amostra é muito diluida, é necessário que a reação se complete, então para aumentar o tempo de residência da amostra no percurso analítico, foram propostos as técnicas de parada de fluxo e fluxo intermitente ${ }^{20,21}$. Muitas vezes, a faixa de concentração das amostras é muito variada, necessitando que algumas sejam analisadas novamente, estabelecendo-se outro tempo de residência no percurso analítico.

O módulo de análise proposto neste trabalho compreende dois percursos analíticos e possibilita que se estebeleça, por software, o intervalo de tempo de residência da amostra em cada um dos percursos analíticos ${ }^{20,21}$.

A vazão do carregador foi estabelecida em $2 \mathrm{~mL} / \mathrm{min}$ e as bobinas de reação foram fixadas em $100 \mathrm{~cm}(0,5 \mathrm{~mL})$, portanto o tempo de trânsito da zona da amostra era $15 \mathrm{~s}$. Em um módulo de análise baseado no processo de amostragem binária, o tempo de trânsito é praticamente igual ao tempo de residência da parte central da zona da amostra, portanto apenas um pequeno percentual da reação se completa até alcançar a cela de fluxo

No módulo de análise da figura 1, o tempo de residência da amostra pode ser maior do que o tempo de trânsito, acionando-se a válvula $\mathrm{V}_{5}$ em intervalos de tempo pré-estabelecidos. Testes preliminares indicaram que um tempo de residência de $50 \mathrm{~s}$, possibilitava que aproximadamente $50 \%$ da reação se completasse, sendo suficiente para trabalhar com amostras na faixa de 1 a $10 \mathrm{mg} / \mathrm{L}$ de fosfato. Este intervalo de tempo foi mantido constante para o percurso analítico definido pela bobina de reação $\mathrm{B}_{2}$ (Fig.1). No caso do percurso analítico compreendido pela bobina $\mathrm{B}_{2}$, o fluxo era mantido durante $50 \mathrm{~s}$ para garantir que toda zona da amostra fosse descartada, antes que se iniciasse o deslocamento da amostra retida em $B_{1}$. Nestas condições, o sinal analítico correspondia aproximadamente a $50 \%$ do valor obtido quando a reação se completava. Manteve-se fixo este valor para a bobina $\mathrm{B}_{2} \mathrm{e}$ variou-se os tempos de residência em $B_{1}$ de 50 até $160 \mathrm{~s}$ e os resultados são mostrados na figura 4 . 


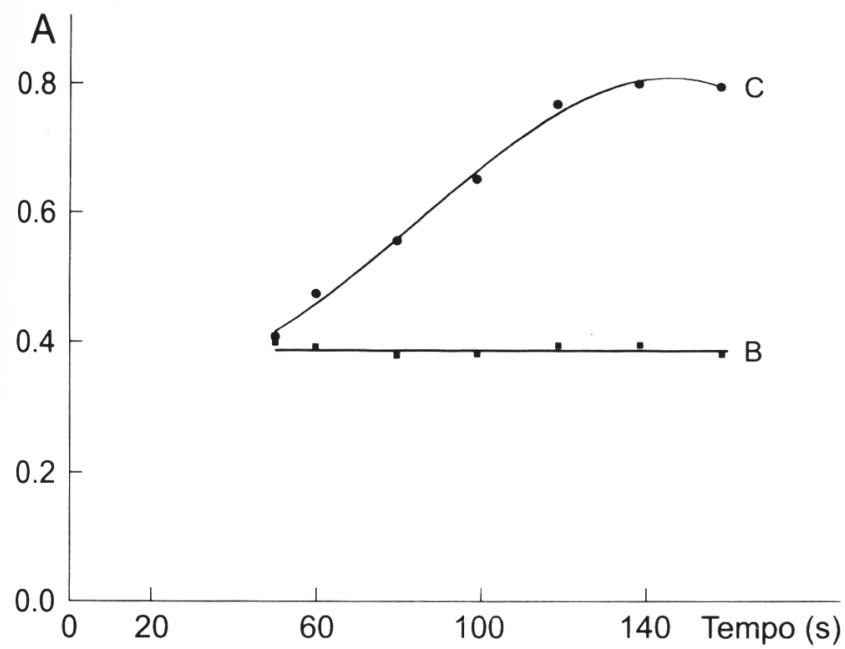

Figura 4. Efeito do tempo de residência da amostra na magnitude do sinal. $A=$ absorbância. Curva $B$ obtida com a bobina de reação $B_{2}$ (Fig.1) com tempo de residência de 50 s. Curva $C$ obtida com a bobina de reação $B_{I}$ variando-se o tempo de residência. Vazão $=33,3$ $\mu \mathrm{L} / \mathrm{s}$; tempos de amostragem $=0,4 \mathrm{~s}$ para as soluçóes da amostra $e$ do molibdato de amônio; e 0,2 s para a solução de ácido ascórbico. Foi usada uma solução $10 \mathrm{mg} / \mathrm{L}$ do ortofosfato.

Analisando-se esta figura, observa-se que no intervalo de 50 a $140 \mathrm{~s}$, obteve-se uma resposta praticamente linear em função do tempo, sendo que a magnitude do sinal analítico teve um aumento em torno de $100 \%$. Portanto, podese aproveitar este recurso para ajustar a magnitude do sinal à faixa operacional do instrumento em função da concentração das amostras.

Nos sistemas FIA usuais, em geral, o reagente é adicionado à zona da amostra por confluência, enquanto no sistema proposto as alíquotas de amostra e do reagente são inseridas sequencialmente, portanto as condições de mistura são completamente distintas. As bobinas de reação $\mathrm{B}_{1}$ e $\mathrm{B}_{2}$ tinham diâmetro interno de $0,8 \mathrm{~mm}$, assim cada microlitro de solução ocupava um espaço de $2 \mathrm{~mm}$. Portanto, o volume das alíquotas da amostra e dos reagentes pode ter um efeito marcante sobre a homogeneização da mistura, afetando as condições de reação e a estabilidade do sinal analítico. O melhor resultado foi encontrado com tempos de amostragem de 0,$5 ; 0,5 ; 0,2 \mathrm{~s}$ para amostra e para os reagentes $R_{1}$ e $R_{2}$, respectivamente. A vazão de bombeamento foi fixada em $33,3 \mu \mathrm{L} / \mathrm{s}$, então, em cada ciclo de acionamento das válvulas $\mathrm{V}_{1}, \mathrm{~V}_{2}, \mathrm{~V}_{3}$, e $\mathrm{V}_{4}$, alíquotas de soluções com volumes de $16,7 \mu \mathrm{L}$ da amostra e do reagente $R_{1}$ e de $6,7 \mu \mathrm{L}$ do reagente $R_{2}$, eram inseridos no percurso analítico $\left(\mathrm{B}_{1}\right.$ ou $\left.\mathrm{B}_{2}\right)$.

Para amostras tendo concentrações na faixa de 0,1 a 1,0 $\mathrm{mg} / \mathrm{L}$, o intervalo de tempo de residência no percurso analítico foi estabelecido em $120 \mathrm{~s}$ e foram programados 12 ciclos consecutivos de amostragem. Assim, o volume da zona de amostra foi de $481 \mu \mathrm{L}$, preenchendo praticamente toda a bobina de reação $(500 \mu \mathrm{L})$. Com este recurso, a perda de sinal causada pela dispersão da amostra no carregador foi minimizada. Para a faixa de concentração de 1 a $10 \mathrm{mg} / \mathrm{L}$, diminuiu-se pela metade o volume da zona da amostra $(240 \mu \mathrm{L})$, programando-se 6 ciclos de amostragem, sendo que o respectivo tempo de residência foi mantido em $50 \mathrm{~s}$ na bobina $\mathrm{B}_{2}$.

No presente trabalho, para amostras na faixa de concentrações de 0,1 a 1,0 mg/L, a zona da amostra compreendia um volume de $480 \mu \mathrm{L}$ ( $200 \mu \mathrm{l}$ de amostra, $200 \mu \mathrm{L}$ de molibdato de amônio e $80 \mu \mathrm{L}$ de ácido ascórbico) e era inserida no percurso analítico em um intervalo de tempo de 14,4 s. Assim, a reação química no interior da primeira alíquota de amostra inserida no percurso analítico, estava se desenvolvendo há $14 \mathrm{~s}$, quando a etapa de amostragem era encerrada. Em um sistema
FIA usual, somente após a injeção, o reagente é adicionado à amostra. Considerando-se o intervalo de tempo para encher a alça de amostragem, então se a amostra tivesse um tempo de residência de $120 \mathrm{~s}$, o período de análise seria superior a $130 \mathrm{~s}$. Deve-se considerar que a alça de amostragem precisa ser lavada com a solução da amostra e, que para alcançar alta sensibilidade, o comprimento deve ser igual ou maior que o do percurso analítico.

Uma vez definidos os parâmetros de operação das duas faixas de concentrações, foi determinado o íon ortofosfato em um conjunto de amostras de águas do rio Piracicaba e de seu afluente, o rio Piracicamirim. Os resultados são mostrados na tabela 1. A exatidão foi averiguada empregando o método FIA usual $^{22}$, e aplicando-se o teste-t pareado, não se verificou diferença significativa ao nível de $95 \%$ de confiabilidade. Também, foram conseguidas outras características analíticas importantes tais como: velocidade analítica de 60 determinações por hora; consumo de reagentes por determinação, $200 \mu \mathrm{L}$ de molibdato de amônio e $80 \mu \mathrm{L}$ ácido ascórbico; e desvio padrao relativo de $2,0 \%(n=12)$. Para amostras na faixa de concentração de 1 a $10 \mathrm{mg} / \mathrm{L}$, o consumo destes reagentes diminuem pela metade.

Tabela 1. Determinação de ortofosfato em amostras de água.

\begin{tabular}{ccc}
\hline Amostra & $\begin{array}{c}\text { Sistema proposto } \\
\left(\mathrm{mg} \mathrm{PO}_{4}{ }^{3-} / \mathrm{L}\right)\end{array}$ & $\begin{array}{c}\mathrm{FIA} \mathrm{usual}^{*} \\
\left(\mathrm{mg} \mathrm{PO}_{4}{ }^{3-} / \mathrm{L}\right)\end{array}$ \\
\hline 01 & $0,21 \pm 0,03$ & $0,24 \pm 0,02$ \\
02 & $0,07 \pm 0,01$ & $0,08 \pm 0,01$ \\
03 & $0,11 \pm 0,04$ & $0,08 \pm 0,03$ \\
04 & $0,80 \pm 0,05$ & $0,81 \pm 0,04$ \\
05 & $0,51 \pm 0,09$ & $0,42 \pm 0,07$ \\
06 & $1,65 \pm 0,16$ & $1,77 \pm 0,09$ \\
07 & $1,34 \pm 0,03$ & $1,29 \pm 0,02$ \\
08 & $2,61 \pm 0,06$ & $2,58 \pm 0,04$ \\
09 & $3,57 \pm 0,38$ & $3,16 \pm 0,09$ \\
10 & $4,17 \pm 0,13$ & $4,28 \pm 0,10$ \\
\hline
\end{tabular}

* Ref. 22.

\section{AGRADECIMENTOS}

Os autores agradecem à FAPESP, à CAPES e ao CNPq pelo apoio fianceiro recebido.

\section{REFERENCIAS}

1. Ruzicka, J; Hansen, E. H.; Flow Injection Analysis, $2^{\text {nd }}$ Ed., John Wiley e Sons, New York, 1988.

2. Ruzicka, J; Analyst 1994, 119, 1925.

3. Kuban, V.; Critical Reviews in Anal. Chem. 1992, 23, 15.

4. Karlber, B.; Thelander, S,; Anal. Chim. Acta 1978, 98, 1.

5 Bergamin, H. F ${ }^{\circ}$; Reis, B. F.; Jacintho, A. O.; Zagatto, E.A.G.; Anal. Chim. Acta 1980, 117, 81.

6. Zagatto, E. A. G.; Reis, B. F.; Bergamin, H. Fo; Krug, F. J.; Anal. Chim. Acta 1979, 109, 45.

7. Reis, B. F.; Jacintho, A. O.; Mortatti J.; Krug, F. J.; Zagatto E. A. G.; Bergamin, H. F ${ }^{\circ}$; Pessenda, L. C. R.; Anal. Chim. Acta 1981, 123, 221.

8. Saki, T.; Ohno N.; J. Flow Anal. 1993, 10, 2.

9. Ruzicka, J.; Hansen, E. H.; Anal. Chim. Acta 1975, 78, 145.

10. Ruzicka, J.; Stewart, W. B.; Zagatto, E. A. G.; Anal. Chim. Acta 1976, 81, 387.

11. Ruzicka, J.; Hansen, E. H.; Mosback, E. A.; Krug, F. J.; Anal. Chem. 1977, 49, 1958.

12. Muraki, H.; Higuchi, K.; Sasaki, M.; Korenagi, T.; Tôei, K.; Anal. Chim. Acta 1992, 261, 345 
13. Reis, B. F.; Bergamin, H. F'; Quim. Nova 1993, 16, 570.

14. Reis, B. F.; Giné, M. F.; Zagatto, E. A. G.; Lima, J. L. F. C.; Lapa, R. A. Anal. Chim. Acta 1994, 293, 129.

15. Martelli, P. B.; Reis B. F.; Kronka, A. M., Korn, M.; Bergamin H. F ${ }^{o}$, Zagatto, E. A. G.; Lima, J. L. F. C.; Araujo, A.; Anal. Chim. Acta 1995, 308, 397.

16. Krug, F. J.; Reis B .F.; Giné, M. F.; Zagatto, E. A. G.; Ferreira, J. R.; Jacintho, A. O.; Anal. Chim. Acta 1983, 151, 39.

17. Reis, B. F.; Giné M. F.; Kronka, E. A. M.; Quim. Nova 1989, 12,82 .
18. Drummond, L.; Maher, W.; Anal. Chim. Acta 1995, 302, 69.

19. Murphy, J.; Riley, J. P.; Anal. Chim. Acta 1962, 27, 26. 20. Christian, G. D.; Ruzicka, J.; Anal. Chim. Acta 1992, $261,11$.

21. Schweizer, B.; Muller, H.; Bohme, W.; Fres. J. Anal. Chem. 1992, 343, 183.

22. Jacintho, A. O.; Kronka, E. A. M.; Zagatto, E. A. G.; Arruda, M. A. Z.; Ferreira, J. R.; J. Flow Injection Anal. 1989, 6,1 . 